\title{
On $V_{n}$-Arithmetic Graph
}

\author{
R. Rangarajan \\ Professor \\ Department of Studies in Mathematics \\ University of Mysore \\ Mysore 570 006, India
}

\author{
Akram Alqesmah \\ Department of Studies in Mathematics \\ University of Mysore \\ Mysore 570 006, India
}

\author{
Anwar Alwardi \\ Department of Studies in Mathematics \\ University of Mysore \\ Mysore 570 006, India
}

\begin{abstract}
$V_{n}$-Arithmetic graph has been introduced by Vasumathi and Vangipuram [9]. In this paper some properties of $V_{n}$-Arithmetic graph, maximum degree, minimum degree, number of edges, diameter, radius, Hamiltonian and Eulerian are studied. Also, we introduce $m$-Arithmetical graphs. Some properties and interesting results for $m$-Arithmetical graphs are established.
\end{abstract}

\section{Keywords}

$V_{n}$-Arithmetic graph, $m$-Arithmetic graph

\section{INTRODUCTION}

Number Theory is one of the oldest branches of mathematics, which inherited rich contributions from almost all greatest mathematicians, ancient and modern. Nathanson [5] was the pioneer in introducing the concepts of Number Theory, particularly, the Theory of Congruences in Graph Theory, and paved the way for the emergence of a new class of graphs, namely Arithmetic Graphs. Inspired by the interplay between Number Theory and Graph Theory several researchers in recent times are carrying out extensive studies on various Arithmetic graphs in which adjacency between vertices is defined through various arithmetic functions.

All the graphs considered here are finite and undirected with no loops and multiple edges. Let $G=(V, E)$ be a graph. As usual $|V|$ and $|E|$ denote the number of vertices and edges of a graph $G$, respectively. In general, we use $\langle X\rangle$ to denote the subgraph induced by the set of vertices $X$ and $N(v)$ and $N[v]$ denote the open neighbourhood and closed neighbourhood of a vertex $v$, respectively. The degree of a vertex $v$, in a graph $G$, is denoted $\operatorname{deg}(v)$, and is defined to be the number of edges incident with $v$. In simple graphs, $\operatorname{deg}(u)=|N(u)|$. The minimum degree of a graph $G$ is denoted by $\delta$, and the maximum degree is denoted by $\Delta$. If $\delta=\Delta=r$ for any graph $G$, we say $G$ is a regular graph of degree $r$.

The distance $d(u, v)$ between any two vertices $u, v \in G$ is the minimum length of a $u-v$ path, and the eccentricity of a vertex $v$ of a connected graph $G$ is $e(v)=\max \{d(u, v), v \in V\}$. The diameter of $G$ is $\operatorname{diam}(v)=\max \{e(v), v \in V\}$ and the radius of $G$ is $\operatorname{rad}(v)=\min \{e(v), v \in V\}$. A cycle passing through all the vertices of a graph is called a Hamiltonian cycle. A graph containing a Hamiltonian cycle is called a Hamiltonian graph. Also, A closed walk in a graph $G$ containing all the edges of $G$ is called an Euler line in $G$. A graph containing an Euler line is called an Euler graph.
All the definitions in this paper are referenced by [1].

Vasumathi and Vangipuram [9] introduced the concept of $V_{n}$ Arithmetic graphs and studied some of its properties. Let $n$ be a positive integer such that $n=p_{1}^{\alpha_{1}} p_{2}^{\alpha_{2}} \ldots p_{k}{ }^{\alpha_{k}}$. Then the $V_{n^{-}}$ Arithmetic graph is defined as the graph whose vertex set consists of the divisors of $n$ and two vertices $u, v$ are adjacent in $V_{n}$ graph if and only if $\operatorname{gcd}(u, v)=p_{i}$ for some prime divisor $p_{i}$ of $n$. In this graph vertex 1 becomes an isolated vertex. Hence we consider $V_{n}$-Arithmetic graph without vertex 1 as the contribution of this isolated vertex is nothing when the properties of these graphs and enumeration of some domination parameters are studied. Clearly, $V_{n}$ graph is a connected graph. Because if $n$ is a prime, then $V_{n}$ graph consists of a single vertex. Hence it is a connected graph. In other cases, by the definition of adjacency in $V_{n}$ there exist edges between prime number vertices and their prime power vertices and also to their prime product vertices. Therefore each vertex of $V_{n}$ is connected to some vertex in $V_{n}$. Some domination parameters and domination parameters of direct product graphs of Cayley graphs with Arithmetic graphs are presented in [6, 7, 4, 8, 3, 2].

In this paper, we obtain some properties of $V_{n}$-Arithmetic graph, maximum degree, minimum degree, number of edges, diameter, radius, Hamiltonian and Eulerian. Also, we introduce $m$ Arithmetical graphs. Some properties and interesting results for $m$ Arithmetical graphs are established.

\section{SOME PROPERTIES OF $V_{N}$-ARITHMETIC GRAPH}

In this section some properties of $V_{n}$-Arithmetic graph are obtained.

Proposition 1. Let $G$ be a $V_{n}$-Arithmetic graph, where $n=$ $p_{1}^{\alpha_{1}} p_{2}^{\alpha_{2}} \ldots p_{k}{ }^{\alpha_{k}}$. Then the number of vertices of $G$ is

$$
|V|=\prod_{i=1}^{k}\left(\alpha_{i}+1\right)-1 .
$$

PROOF. Straightforward by Fundamental Theorem of Arithmetic, the number of positive divisors $d(n)$ of any natural number $n=p_{1}^{\alpha_{1}} p_{2}^{\alpha_{2}} \ldots p_{k}^{\alpha_{k}}$ is

$$
d(n)=\prod_{i=1}^{k}\left(\alpha_{i}+1\right),
$$


then, by the definition of $V_{n}$-Arithmetic graph

$$
|V|=\prod_{i=1}^{k}\left(\alpha_{i}+1\right)-1
$$

THEOREM 1. Let $G$ be a $V_{n}$-Arithmetic graph, where $n=$ $p_{1}^{\alpha_{1}} p_{2}^{\alpha_{2}} \ldots p_{k}^{\alpha_{k}}$. For any vertex $u=\prod_{i \in B} p_{i}^{a_{i}}$, where $B \subseteq$ $\{1,2, \ldots, k\}, 1 \leq a_{i} \leq \alpha_{i}, \forall i \in B$,

(1) If $u=p_{j}$, where $j \in\{1,2, \ldots, k\}$, then

$$
\operatorname{deg}(u)=\alpha_{j} \prod_{\substack{i=1 \\ i \neq j}}^{k}\left(\alpha_{i}+1\right)-1
$$

(2) If $u=\prod_{i \in B} p_{i}^{a_{i}}, 1<a_{i} \leq \alpha_{i}, \forall i \in B$, then

$$
\operatorname{deg}(u)=|B| \prod_{\substack{i=1 \\ i \notin B}}^{k}\left(\alpha_{i}+1\right) .
$$

(3) If $u=\prod_{i \in B} p_{i}^{a_{i}}, a_{i}=1$ for some $i \in B^{\prime} \subseteq B$, then

$$
\operatorname{deg}(u)=\left(\left|B-B^{\prime}\right|+\sum_{i \in B^{\prime}} \alpha_{i}\right) \prod_{\substack{i=1 \\ i \notin B}}^{k}\left(\alpha_{i}+1\right) .
$$

Proof. Let $u=\prod_{i \in B} p_{i}^{a_{i}}$ be a vertex of $G$, where $B \subseteq$ $\{1,2, \ldots, k\}, 1 \leq a_{i} \leq \alpha_{i}, \forall i \in B$. Since, $|V|=\prod_{i=1}^{k}\left(\alpha_{i}+\right.$ $1)-1$, then the degree of $u \in V(G)$ is the number of vertices in $G$ minus the number of the vertices which are not adjacent to $u$, so, Case 1. Let $u=p_{j}, j \in\{1,2, \ldots, k\}$. Then

$$
\begin{gathered}
\operatorname{deg}(u)=\left(\prod_{i=1}^{k}\left(\alpha_{i}+1\right)-1\right)-\left(\prod_{\substack{i=1 \\
i \neq j}}^{k}\left(\alpha_{i}+1\right)-1\right)-1 \\
=\alpha_{j} \prod_{\substack{i=1 \\
i \neq j}}^{k}\left(\alpha_{i}+1\right)-1 .
\end{gathered}
$$

Case 2. Let $u=\prod_{i \in B} p_{i}^{a_{i}}, 1<a_{i} \leq \alpha_{i}, \forall i \in B$. Suppose that, $B=\{1,2, \ldots, r\}, r \leq k$ (elements of $B$ need not be ordered). Then

$$
\begin{aligned}
& \operatorname{deg}(u)=\left(\prod_{i=1}^{k}\left(\alpha_{i}+1\right)-1\right)-\left(\prod_{\substack{i=1 \\
i \notin B}}^{k}\left(\alpha_{i}+1\right)-1\right) \\
& -\left(\sum_{i=1}^{r}\left(\alpha_{i}-1\right)\right) \prod_{\substack{i=1 \\
i \notin B}}^{k}\left(\alpha_{i}+1\right)-\left(\sum_{i=1}^{r-1} \alpha_{i} \sum_{j=i+1}^{r} \alpha_{j}\right) \\
& \prod_{\substack{i=1 \\
i \notin B}}^{k}\left(\alpha_{i}+1\right)-\left(\sum_{i=1}^{r-2} \alpha_{i} \sum_{j=i+1}^{r-1} \alpha_{j} \sum_{l=j+1}^{r} \alpha_{l}\right) \prod_{\substack{i=1 \\
i \notin B}}^{k}\left(\alpha_{i}+1\right)
\end{aligned}
$$

$$
-\ldots-\left(\prod_{i=1}^{r} \alpha_{i}\right) \prod_{\substack{i=1 \\ i \notin B}}^{k}\left(\alpha_{i}+1\right)=|B| \prod_{\substack{i=1 \\ i \notin B}}^{k}\left(\alpha_{i}+1\right) .
$$

Because,

$$
\begin{gathered}
\prod_{i=1}^{r}\left(\alpha_{i}+1\right)=1+\sum_{i=1}^{r} \alpha_{i}+\sum_{i=1}^{r-1} \alpha_{i} \sum_{j=i+1}^{r} \alpha_{j}+\sum_{i=1}^{r-2} \alpha_{i} \\
\sum_{j=i+1}^{r-1} \alpha_{j} \sum_{l=j+1}^{r} \alpha_{l}+\ldots+\prod_{i=1}^{r} \alpha_{i} .
\end{gathered}
$$

Case 3. Let $u=\prod_{i \in B} p_{i}^{a_{i}}$, where $a_{i}=1$ for some $i \in B^{\prime} \subseteq B$. Then, all the vertices of the form $p_{j}^{a} \prod_{\substack{i=1 \\ i \notin B}} p_{i}^{a_{i}}$, where $1 \leq a \leq \alpha_{j}$, $\forall j \in B$ are adjacent to $u$, then

$$
\begin{gathered}
\operatorname{deg}(u)=\prod_{i=1}^{k}\left(\alpha_{i}+1\right)-\prod_{\substack{i=1 \\
i \notin B}}^{k}\left(\alpha_{i}+1\right)-\left(\sum _ { i \in B - B ^ { \prime } } \left(\alpha_{i}-1\right.\right. \\
\prod_{\substack{i=1 \\
i \notin B}}^{k}\left(\alpha_{i}+1\right)-\left(\sum_{i=1}^{r-1} \alpha_{i} \sum_{j=i+1}^{r} \alpha_{j}\right) \prod_{\substack{i=1 \\
i \notin B}}^{k}\left(\alpha_{i}+1\right) \\
-\left(\sum_{i=1}^{r-2} \alpha_{i} \sum_{j=i+1}^{r-1} \alpha_{j} \sum_{l=j+1}^{r} \alpha_{l}\right) \prod_{\substack{i=1 \\
i \notin B}}^{k}\left(\alpha_{i}+1\right) \\
-\ldots-\left(\prod_{i=1}^{r} \alpha_{i}\right) \prod_{\substack{i=1 \\
i \notin B}}^{k}\left(\alpha_{i}+1\right) \\
=\left(\left|B-B^{\prime}\right|+\sum_{i \in B^{\prime}} \alpha_{i}\right) \prod_{\substack{i=1 \\
i \notin B}}^{k}\left(\alpha_{i}+1\right) .
\end{gathered}
$$

From Theorem 1. it is easy to see that for any two vertices $u, v$ of $G$ such that $u=\prod_{i=1}^{j} p_{i}^{a_{i}}, v=\prod_{i=1}^{j} p_{i}^{b_{i}}, j \leq k$ (the elements of $\{1,2, \ldots, j\}$ need not be ordered), then $\operatorname{deg}(u)=\operatorname{deg}(v)$, where $1<a_{i} \leq \alpha_{i}, 1<b_{i} \leq \alpha_{i}, \forall i \in\{1,2, \ldots, j\}$. Let $G$ be a $V_{n^{-}}$ Arithmetic graph, where $n=p_{1}^{\alpha_{1}} p_{2}^{\alpha_{2}} \ldots p_{k}{ }^{\alpha_{k}}$. Then,

$|V|= \begin{cases}\operatorname{deg}\left(p_{j}\right)+\operatorname{deg}\left(p_{j}^{a}\right), & \text { if at least } \alpha_{j} \neq 1 \text { and } 1<a \leq \alpha_{j} \\ \operatorname{deg}\left(p_{j}\right)+\operatorname{deg}\left(p_{r} p_{s}\right), & \text { if } \alpha_{i}=1, \forall i \in\{1,2, \ldots, k\} .\end{cases}$

Proof. Let $G$ be a $V_{n}$-Arithmetic graph, where $n=$ $p_{1}^{\alpha_{1}} p_{2}^{\alpha_{2}} \ldots p_{k}^{\alpha_{k}}$.

Case1. Suppose that, at least $\alpha_{j} \neq 1$ and $1<a \leq \alpha_{j}$. Then

$\operatorname{deg}\left(p_{j}\right)+\operatorname{deg}\left(p_{j}^{a}\right)=\alpha_{j} \prod_{\substack{i=1 \\ i \neq j}}^{k}\left(\alpha_{i}+1\right)-1+\prod_{\substack{i=1 \\ i \neq j}}^{k}\left(\alpha_{i}+1\right)=|V|$. 
Case2. Suppose that, $\alpha_{i}=1, \forall i \in\{1,2, \ldots, k\}$. Then the number of vertices of $G$ in this case is $|V|=2^{k}-1$ and so,

$$
\operatorname{deg}\left(p_{j}\right)+\operatorname{deg}\left(p_{r} p_{s}\right)=2^{k-1}-1+2^{k-1}=|V| .
$$

THEOREM 2. Let $G$ be a $V_{n}$-Arithmetic graph, where $n=$ $p_{1}^{\alpha_{1}} p_{2}^{\alpha_{2}} \ldots p_{k}{ }^{\alpha_{k}}$, such that at least one of $\alpha_{i}, i \in\{1,2, \ldots, k\}$ does not equal one. Then,

(1) $\Delta(G)=\alpha_{j} \prod_{\substack{i=1 \\ i \neq j}}^{k}\left(\alpha_{i}+1\right)-1$,

where, $\alpha_{j}$ is the maximum exponent of $p_{i}, i \in\{1,2, \ldots, k\}$;

(2) $\delta(G)=k$.

Proof. Let $n=p_{1}^{\alpha_{1}} p_{2}^{\alpha_{2}} \ldots p_{k}{ }^{\alpha_{k}}$ such that at least one of $\alpha_{i}$, $i \in\{1,2, \ldots, k\}$ does not equal one. Then, we have the following cases:

(1) Suppose that, $u=p_{j}$ for some $\left.j \in\{1,2, \ldots, k\}\right)$ be a vertex of $G$ such that $\alpha_{j}$ is the maximum exponent of $p_{i}$, $i \in\{1,2, \ldots, k\}$. The vertex $u=p_{j}$ has the maximum degree of $G$ which is

$$
\operatorname{deg}(u)=\alpha_{j} \prod_{\substack{i=1 \\ i \neq j}}^{k}\left(\alpha_{i}+1\right)-1 .
$$

By Theorem 1 the vertex $u=p_{j}$ has the greatest degree among the primes and the power primes vertices of $G$. Furthermore Theorem 1 tell us the vertices of the form $p_{i} p_{j}, i \neq j$, $(i, j \in\{1,2, \ldots, k\})$ have degrees greater than the degrees of all the primes product vertices, which is smaller than or equal the degree of $u=p_{j}$, because, either,

$$
\begin{gathered}
\operatorname{deg}\left(p_{j}\right)-\operatorname{deg}\left(p_{j} p_{l}\right)=\left(\alpha_{j} \prod_{\substack{i=1 \\
i \neq j}}^{k}\left(\alpha_{i}+1\right)-1\right) \\
-\left(\alpha_{j}+\alpha_{l}\right) \prod_{\substack{i=1 \\
i \neq j, l}}^{k}\left(\alpha_{i}+1\right) \\
=-1+\alpha_{l}\left(\alpha_{j}-1\right) \prod_{\substack{i=1 \\
i \neq j, l}}^{k}\left(\alpha_{i}+1\right) \geq 0
\end{gathered}
$$

since, $\alpha_{j}>1$.

or,

$$
\begin{aligned}
\operatorname{deg}\left(p_{j}\right)- & \operatorname{deg}\left(p_{r} p_{s}\right)=\left(\alpha_{j} \prod_{\substack{i=1 \\
i \neq j}}^{k}\left(\alpha_{i}+1\right)-1\right) \\
& -\left(\alpha_{r}+\alpha_{s}\right) \prod_{\substack{i=1 \\
i \neq r, s}}^{k}\left(\alpha_{i}+1\right)
\end{aligned}
$$

$$
=-1+\left(\left(\alpha_{r} \alpha_{s}+1\right)\left(\alpha_{j}+1\right)-\left(\alpha_{r}+1\right)\left(\alpha_{s}+1\right)\right) \prod_{\substack{i=1 \\ i \neq j, r, s}}^{k}\left(\alpha_{i}+1\right)
$$

since, all of $\alpha_{i}, i \in\{1,2, \ldots, k\}$ are positive integers and $\alpha_{j}>1$ is the greatest value of them then,

$$
\left(\alpha_{r} \alpha_{s}+1\right)\left(\alpha_{j}+1\right)-\left(\alpha_{r}+1\right)\left(\alpha_{s}+1\right) \geq 1
$$

so,

$$
\operatorname{deg}\left(p_{j}\right)-\operatorname{deg}\left(p_{r} p_{s}\right) \geq 0 .
$$

Which leads to the required result.

(2) On the other hand, the vertex $t=\prod_{i=1}^{k} p_{i}^{\alpha_{i}}$ has the minimum degree of $G$ which is $\operatorname{deg}(t)=\stackrel{\bar{k}}{ }$ (Theorem 1). Suppose that, $u=\prod_{i=1}^{j} p_{i}^{a_{i}}, j<k$ (elements of $\{1,2, \ldots, j\}$ need not be ordered) be a vertex of $G$, where $1 \leq a_{i} \leq \alpha_{i}$, $\forall i \in\{1,2, \ldots, j\}$. Then,

$$
\begin{aligned}
\operatorname{deg}(u)> & j+\left(\begin{array}{l}
j \\
1
\end{array}\right)\left[\left(\begin{array}{c}
k-j \\
1
\end{array}\right)+\left(\begin{array}{c}
k-j \\
2
\end{array}\right)+\ldots\right. \\
& \left.+\left(\begin{array}{c}
k-j \\
k-j
\end{array}\right)\right]>j 2^{k-j} \geq k .
\end{aligned}
$$

Also, if $j=k$, then by Theorem $1 \operatorname{deg}(u) \geq k$.

THEOREM 3. Let $G$ be a $V_{n}$-Arithmetic graph, where $n=$ $p_{1} p_{2} \ldots p_{k}\left(\alpha_{i}=1, \forall i \in\{1,2, \ldots, k\}\right)$. Then,

(1) $\Delta(G)=2^{k-1}$

(2) $\delta(G)= \begin{cases}k, & k \geq 3 \\ 1, & k=2\end{cases}$

Proof. Let $n=p_{1} p_{2} \ldots p_{k}$. By substitute $\alpha_{i}=1$, $\forall i \in\{1,2, \ldots, k\}$ in Theorem 1 we get for any $u \in G$,

$$
\operatorname{deg}(u)= \begin{cases}2^{k-1}-1, & \text { if } u=p_{j}, \text { for some } j \in\{1,2, \ldots, k\} \\ |B| 2^{k-|B|}, & \text { if } u=\prod_{i \in B} p_{i} .\end{cases}
$$

We observe that when $|B|=2$, then the vertices $u=p_{i} p_{j}, i, j \in$ $\{1,2, \ldots, k\}$ have the maximum degree of $G$ which is

$$
\Delta(G)=\operatorname{deg}(u)=2^{k-1}
$$

Also, the minimum degree of $G$,

$$
\delta(G)=\operatorname{deg}\left(\prod_{i=1}^{k} p_{i}\right)=k,
$$

where $k \geq 3$. But, if $k=2$ (i.e., $n=p_{1} p_{2}$ ), then $V=$ $\left\{p_{1}, p_{2}, p_{1} p_{2}\right\}$. So, $\operatorname{deg}\left(p_{1}\right)=1, \operatorname{deg}\left(p_{2}\right)=1$ and $\operatorname{deg}\left(p_{1} p_{2}\right)=$ 2 , hence, $\delta(G)=1$.

The connectivity $\kappa=\kappa(G)$ of a graph $G$ is the minimum number of vertices whose removal results in a disconnected graph. For $\kappa \geq$ $k$, we say that $G$ is $k$-connected. By other words, a $k$-connected graph is the graph that the removal of fewer than $k$ vertices will not disconnect it.

THEOREM 4. Let $G$ be a $V_{n}$-Arithmetic graph, where $n=$ $p_{1}^{\alpha_{1}} p_{2}^{\alpha_{2}} \ldots p_{k}^{\alpha_{k}}$ such that $n \neq p_{1} p_{2}$. Then $G$ is a $k$-connected graph. 
Proof. Let $G$ be a $V_{n}$-Arithmetic graph, where $n=$ $p_{1}^{\alpha_{1}} p_{2}^{\alpha_{2}} \ldots p_{k}^{\alpha_{k}}$ such that $n \neq p_{1} p_{2}$. Then from Theorem 2 and Theorem 3 the minimum degree of $G$ is $\delta(G)=k$, which is the degree of the vertices of the form $t=\prod_{i=1}^{k} p_{i}^{a_{i}}$, where $1<a_{i} \leq \alpha_{i}$, $\forall i \in\{1,2, \ldots, k\}$ (or $t=\prod_{i=1}^{k} p_{i}$ if $\alpha_{i}=1, \forall i \in\{1,2, \ldots, k\}$ ) because, those vertices adjacent only to the prime vertices $p_{i}$, $i \in\{1,2, \ldots, k\}$ and each the other vertices have degrees greater than or equal $k$. So, the minimum number of vertices whose removal disconnect the graph $G$ is $k$. Hence, $G$ is a $k$-connected graph.

By using the Euler Theorem, the number of edges of any graph $G(V, E)$ is given by:

$$
|E|=\frac{1}{2} \sum_{i=1}^{n} \operatorname{deg}\left(u_{i}\right), \quad \forall u_{i} \in V .
$$

Proposition 2. Let $G$ be a $V_{n}$-Arithmetic graph, where $n=$ $p_{1}^{\alpha_{1}} p_{2}^{\alpha_{2}} \ldots p_{k}^{\alpha_{k}}$. Then

$$
\begin{gathered}
|E|=\frac{1}{2}\left(\sum_{i=1}^{k} \operatorname{deg}\left(p_{i}\right)+\sum_{i=1}^{k-1} \sum_{j=i+1}^{k} \operatorname{deg}\left(p_{i} p_{j}\right)+\right. \\
\sum_{i=1}^{k-2} \sum_{j=i+1}^{k-1} \sum_{l=j+1}^{k} \operatorname{deg}\left(p_{i} p_{j} p_{l}\right)+\ldots+\operatorname{deg}\left(\prod_{i=1}^{k} p_{i}\right)+ \\
k \sum_{j=1}^{k}\left(\alpha_{j}-1\right) \operatorname{deg}\left(p_{j}^{a}\right)+\sum_{j=1}^{k}\left(\alpha_{j}-1\right) \sum_{\substack{l=1 \\
l \neq j}}^{k} \sum_{\substack{m=1 \\
m \neq j, l}}^{k} \operatorname{deg}\left(p_{j}^{a} p_{l} p_{m}\right) \\
+\ldots+\sum_{j=1}^{k}\left(\alpha_{j}-1\right) \operatorname{deg}\left(p_{j}^{a} \prod_{\substack{i=1 \\
i \neq j}}^{k}+\sum_{j=1}^{k-1}\left(\alpha_{j}-1\right) \sum_{\substack{l=j+1 \\
j=g}}^{k}\left(\alpha_{l}-1\right)\right. \\
+\ldots+\sum_{j=1}^{k}\left(\alpha_{j}-1\right) \sum_{\substack{k=j+1 \\
l}}^{k}\left(\alpha_{l}-1\right) \operatorname{deg}\left(p_{j}^{a} p_{l}^{b} \prod_{\substack{i=1 \\
i \neq j, l}}^{k} p_{i}\right) \\
+\sum_{j=1}^{k-1}\left(\alpha_{j}-1\right) \sum_{\substack{l=j+1 \\
k=1}}^{k}\left(\alpha_{l}-1\right) \sum_{\substack{m=1 \\
m \neq j, l}}^{k} \operatorname{deg}\left(p_{j}^{a} p_{l}^{b} p_{m}\right) \\
+\sum_{l=j+1}^{k}\left(\alpha_{l}-1\right) \sum_{\substack{m=1 \\
m \neq j, l}}^{k} \sum_{\substack{r=1 \\
m \neq j, l, m}}^{k} \operatorname{deg}\left(p_{j}^{a} p_{l}^{b} p_{m} p_{r}\right)
\end{gathered}
$$

such that, $1<a \leq \alpha_{j}, 1<b \leq \alpha_{l}, \ldots$.

It's clearly that, this formula is very long and difficult to use, and we cannot reduce it to a short formula because the degrees of the vertices of $G$ are depending on the powers $\alpha_{i}$ 's and the options of their products. But we can make a short formula for special cases of $G$.

THEOREM 5. Let $G$ be a $V_{n}$-Arithmetic graph, where $n=$ $p_{1}^{\alpha} p_{2}^{\alpha} \ldots p_{k}^{\alpha}\left(\alpha_{i}=\alpha, \forall i \in\{1,2, \ldots, k\}\right)$. Then the number of edges of $G$ is given by

$|E|=\frac{1}{2}\left[-k+\sum_{j=0}^{k}\left(\begin{array}{l}k \\ j\end{array}\right)(\alpha-1)^{j} \sum_{i=0}^{k-j}\left(\begin{array}{c}k-j \\ i\end{array}\right)(i \alpha+j)(\alpha+1)^{k-i-j}\right]$

Proof. Let $G$ be a $V_{n}$-Arithmetic graph, where $n=$ $p_{1}^{\alpha} p_{2}^{\alpha} \ldots p_{k}^{\alpha}$. Then

$$
|E|=\frac{1}{2} \sum_{i=1}^{(\alpha+1)^{k}-1} \operatorname{deg}\left(u_{i}\right), \quad u_{i} \in V .
$$

So,

$$
\begin{aligned}
& \sum_{i=1}^{(\alpha+1)^{k}-1} \operatorname{deg}\left(u_{i}\right)=\left(\begin{array}{l}
k \\
1
\end{array}\right) \operatorname{deg}\left(p_{j}\right)+\left(\begin{array}{l}
k \\
2
\end{array}\right) \operatorname{deg}\left(p_{j} p_{l}\right)+\ldots+\left(\begin{array}{l}
k \\
k
\end{array}\right) \\
& \operatorname{deg}\left(\prod_{i=1}^{k} p_{i}\right)+\left(\begin{array}{l}
k \\
1
\end{array}\right)\left(\begin{array}{c}
\alpha-1 \\
1
\end{array}\right) \operatorname{deg}\left(p_{j}^{a}\right)+\left(\begin{array}{l}
k \\
1
\end{array}\right)\left(\begin{array}{c}
\alpha-1 \\
1
\end{array}\right)\left(\begin{array}{c}
k-1 \\
1
\end{array}\right) \\
& \operatorname{deg}\left(p_{j}^{a} p_{l}\right)+\left(\begin{array}{c}
k \\
1
\end{array}\right)\left(\begin{array}{c}
\alpha-1 \\
1
\end{array}\right)\left(\begin{array}{c}
k-1 \\
2
\end{array}\right) \operatorname{deg}\left(p_{j}^{a} p_{l} p_{m}\right)+\ldots+\left(\begin{array}{c}
k \\
1
\end{array}\right) \\
& \left(\begin{array}{c}
\alpha-1 \\
1
\end{array}\right)\left(\begin{array}{l}
k-1 \\
k-1
\end{array}\right) \operatorname{deg}\left(p_{j}^{a} \prod_{\substack{i=1 \\
i \neq j}}^{k} p_{i}\right)+\left(\begin{array}{l}
k \\
2
\end{array}\right)\left[\left(\begin{array}{c}
\alpha-1 \\
1
\end{array}\right)\right]^{2} \operatorname{deg}\left(p_{j}^{a} p_{l}^{b}\right) \\
& +\left(\begin{array}{l}
k \\
2
\end{array}\right)\left[\left(\begin{array}{c}
\alpha-1 \\
1
\end{array}\right)\right]^{2}\left(\begin{array}{c}
k-2 \\
1
\end{array}\right) \operatorname{deg}\left(p_{j}^{a} p_{l}^{b} p_{m}\right)+\ldots+\left(\begin{array}{l}
k \\
2
\end{array}\right)\left[\left(\begin{array}{c}
\alpha-1 \\
1
\end{array}\right)\right]^{2} \\
& \left(\begin{array}{l}
k-2 \\
k-2
\end{array}\right) \operatorname{deg}\left(p_{j}^{a} p_{l}^{b} \prod_{\substack{i=1 \\
i \neq j, l}}^{k} p_{i}\right)+\ldots+\left(\begin{array}{c}
k \\
k-1
\end{array}\right)\left[\left(\begin{array}{c}
\alpha-1 \\
1
\end{array}\right)\right]^{k-1} \\
& \operatorname{deg}\left(\prod_{\substack{i=1 \\
i \neq j}}^{k} p_{i}^{a_{i}}\right)+\left(\begin{array}{c}
k \\
k-1
\end{array}\right)\left[\left(\begin{array}{c}
\alpha-1 \\
1
\end{array}\right)\right]^{k-1} \\
& \operatorname{deg}\left(p_{j} \prod_{\substack{i=1 \\
i \neq j}}^{k} p_{i}^{a_{i}}\right)+\left(\begin{array}{l}
k \\
k
\end{array}\right)\left[\left(\begin{array}{c}
\alpha-1 \\
1
\end{array}\right)\right]^{k} \operatorname{deg}\left(\prod_{i=1}^{k} p_{i}^{a_{i}}\right)
\end{aligned}
$$

where, $1<a \leq \alpha_{j}, 1<b \leq \alpha_{l}, \ldots$.

By substitute $\alpha_{i}=\alpha, \forall i \in\{1,2, \ldots, k\}$ in Theorem 1 we get for any vertex $u$ of $G$

(1) $\operatorname{deg}(u)= \begin{cases}\alpha(\alpha+1)^{k-1}-1, & \text { if } u=p_{i}, i \in\{1,2, \ldots, k\} ; \\ |B|(\alpha+1)^{k-|B|}, & \text { if } u=\prod_{i \in B} p_{i}^{a}, 1<a \leq \alpha, \forall i \in B\end{cases}$ 
(2) $\operatorname{deg}(u)=\left(\left|B-B^{\prime}\right|+\left|B^{\prime}\right| \alpha\right)(\alpha+1)^{k-|B|}$, if $u=\prod_{i \in B} p_{i}^{a}$, $a=1$ for some $i \in B^{\prime} \subseteq B$.

Then

$$
\begin{gathered}
\sum_{i=1}^{(\alpha+1)^{k}-1} \operatorname{deg}\left(u_{i}\right)=-k+\sum_{i=0}^{k} i \alpha\left(\begin{array}{c}
k \\
i
\end{array}\right)(\alpha+1)^{k-i}+k(\alpha-1) \\
\sum_{i=0}^{k-1}\left(\begin{array}{c}
k-1 \\
i
\end{array}\right)(i \alpha+1)(\alpha+1)^{k-i-1}+ \\
\left(\begin{array}{c}
k \\
2
\end{array}\right)(\alpha-1)^{2} \sum_{i=0}^{k-2}\left(\begin{array}{c}
k-2 \\
i
\end{array}\right)(i \alpha+2)(\alpha+1)^{k-i-2}+\ldots \\
+\left(\begin{array}{c}
k \\
k-1
\end{array}\right)\left(\begin{array}{c}
\alpha-1)^{k-1}(i \alpha+k-1)(\alpha+1)+k(\alpha-1)^{k} \\
=-k+\sum_{j=0}^{k}\left(\begin{array}{c}
k \\
j
\end{array}\right)(\alpha-1)^{j} \sum_{i=0}^{k-j}\left(\begin{array}{c}
k-j \\
i
\end{array}\right)(i \alpha+j)(\alpha+1)^{k-i-j} .
\end{array}\right.
\end{gathered}
$$

Let $G$ be a $V_{n}$-Arithmetic graph, where $n=p_{1} p_{2} \ldots p_{k}\left(\alpha_{i}=1\right.$, $\forall i \in\{1,2, \ldots, k\})$. Then the number of edges of $G$ is given by

$$
|E|=\frac{1}{2}\left(\sum_{i=1}^{k-1} i\left(\begin{array}{l}
k \\
i
\end{array}\right) 2^{k-i}\right) .
$$

Proposition 3. Let $G$ be a $V_{n}$-Arithmetic graph, where $n=$ $p_{1}^{\alpha_{1}} p_{2}^{\alpha_{2}} \ldots p_{k}^{\alpha_{k}}$. Then, the diameter of $G$ at most equal 2.

Proof. Let $G$ be a $V_{n}$-Arithmetic graph, such that $V(G)=$ $X_{1} \cup X_{2} \cup X_{3}$, where $X_{1}=\left\{p_{i}: i=1,2, \ldots, k\right\}, X_{2}=\left\{p_{i}^{a}\right.$ : $\left.1<a \leq \alpha_{i}, i=1,2, \ldots, k\right\}$ and $X_{3}=V-\left(X_{1} \cup X_{2}\right)$. we have two cases:

Case I. Suppose that, $k>1$ and $n \neq p_{1} p_{2}$. Then, we have the following subcases:

Subcase 1. Assume that, $u, v \in X_{1}$, where $u=p_{i}, v=p_{j}, i \neq j$. Then there is a vertex $t \in X_{3}, t=p_{i} p_{j}$ which adjacent to both $u$ and $v$. So, $d(u, v)=2$.

Subcase 2. Assume that, $u, v \in X_{2}$. Then we have two possibilities:

(1) Let $u$ and $v$ are different powers of the same prime $p_{i}$. Then they are adjacent to the vertex $p_{i} \in X_{1}$. So, $d(u, v)=2$.

(2) Let $u=p_{i}^{a}$ and $v=p_{j}^{b}, i \neq j$. Then there is a vertex $t \in X_{3}$, $t=p_{i} p_{j}$ which adjacent to both $u$ and $v$. So, $d(u, v)=2$.

Subcase 3. Assume that, $u, v \in X_{3}$. Then there are two possibilities:

(1) Let $\operatorname{gcd}(u, v)=\prod_{i \in B} p_{i}^{a_{i}}$ where $B \subseteq\{1,2, \ldots, k\}, 1 \leq$ $a_{i} \leq \alpha_{i}, \forall i \in B$. Then $u$ and $v$ are either adjacent (if $|B|=1$ and $a=1$ ) or choose $r \in B$ such that the vertex $p_{r} \in X_{1}$ is adjacent to both $u$ and $v$. So, $d(u, v)=1$ or $d(u, v)=2$.

(2) Let $\operatorname{gcd}(u, v)=1$. Then there is a vertex $t \in X_{3}, t=p_{r} p_{s}$ such that, $p_{r}$ is a prime divisor of $u$ and $p_{s}$ is a prime divisor of $v$ which is adjacent to both $u$ and $v$. So, $d(u, v)=2$.
Subcase 4. Assume that, $u \in X_{1}$ and $v \in X_{2}$. Then there are two possibilities:

(1) Let $\operatorname{gcd}(u, v)=p_{i}, i \in\{1,2, \ldots, k\}$. Then $d(u, v)=1$.

(2) Let $\operatorname{gcd}(u, v)=1$, i.e. $u=p_{r}, v=p_{s}^{a}$, where $r \neq s$. Then there exists a vertex $t \in X_{3}, t=p_{r} p_{s}$ which is adjacent to both $u$ and $v$. So, $d(u, v)=2$.

Subcase 5. Assume that, $u \in X_{1}$ and $v \in X_{3}$. Then there are two possibilities:

(1) Let $\operatorname{gcd}(u, v)=p_{i}, i \in\{1,2, \ldots, k\}$. Then $d(u, v)=1$.

(2) Let $\operatorname{gcd}(u, v)=1$, i.e. $u=p_{r}, v=\prod_{\substack{i \in B \\ r \notin B}} p_{i}^{a_{i}}, B \subseteq$ $\{1,2, \ldots, k\}, 1 \leq a_{i} \leq \alpha_{i}, \forall i \in B$. Then there exists a vertex $t \in X_{3}, t=p_{r} p_{s}$ such that $p_{s}$ is a prime divisor of $v$ which is adjacent to both $u$ and $v$. So, $d(u, v)=2$.

Subcase 6. Assume that, $u \in X_{2}$ and $v \in X_{3}$. Then we characterize two possibilities:

(1) Let $\operatorname{gcd}(u, v)=p_{r}^{b}, 1 \leq b \leq a \leq \alpha_{r}$. Then $u$ and $v$ are either adjacent (if $b=1$ ) or there is a vertex $p_{r} \in X_{1}$ which is adjacent to both $u$ and $v$. So, $d(u, v)=1$ or $d(u, v)=2$.

(2) Let $\operatorname{gcd}(u, v)=1$, i.e. $u=p_{r}^{a}, v=\prod_{\substack{i \in B \\ r \notin B}} p_{i}^{a_{i}}, B \subseteq$ $\{1,2, \ldots, k\}, 1 \leq a_{i} \leq \alpha_{i}, \forall i \in B$. Then there exists a vertex $t \in X_{3}, t=p_{r} p_{s}$ such that $p_{s}$ is a prime divisor of $v$ which is adjacent to both $u$ and $v$. So, $d(u, v)=2$.

Hence, if $n=p_{1}^{\alpha_{1}} p_{2}^{\alpha_{2}} \ldots p_{k}^{\alpha_{k}}, k>1$ then, $\operatorname{diam}(G)=2$.

Case II. Suppose that, $k=1$ (i.e. $n=p^{\alpha}$ where $p$ is prime) or $n=p_{1} p_{2}$. Then we characterize three subcases:

Subcase 1. Let $\alpha=2 \Rightarrow n=p^{2}$. Then $G$ has only two vertices $p, p^{2}$ and one edge joining $p$ and $p^{2}$. So, $\operatorname{diam}(G)=1$.

Subcase 2. Let $\alpha>2$. Then $X_{1}=\{p\}, X_{2}=\left\{p_{2}, \ldots, p_{\alpha}\right\}$ and $X_{3}=\phi$, so,

(1) For $u, v \in X_{2}$, the vertex $p \in X_{1}$ is adjacent to both $u$ and $v$. So, $d(u, v)=2$.

(2) For $u=p \in X_{1}$ and $v \in X_{2}$, then $\operatorname{gcd}(u, v)=p$ and hence, $d(u, v)=1$.

This means that, $e(u)=2$ for $u \in X_{2}$ and $e(u)=1$ for $u \in X_{1}$. Subcase 3. Let $n=p_{1} p_{2}$. Then $X_{1}=\left\{p_{1}, p_{2}\right\}, X_{3}=\left\{p_{1} p_{2}\right\}$ and $X_{2}=\phi$. So, as in subcase2, $e(u)=2$ for $u \in X_{1}$ and $e(u)=1$ for $u \in X_{3}$.

Let $G$ be a $V_{n}$-Arithmetic graph, where $n=p_{1}^{\alpha_{1}} p_{2}^{\alpha_{2}} \ldots p_{k}{ }^{\alpha_{k}}$ such that, $k>1$ and $n \neq p_{1} p_{2}$. Then

$$
\operatorname{diam}(G)=2=\operatorname{rad}(G) .
$$

THEOREM 6. Let $G$ be a $V_{n}$-Arithmetic graph, where $n=$ $p_{1}^{\alpha_{1}} p_{2}^{\alpha_{2}} \ldots p_{k}^{\alpha_{k}}$. Then $G$ is not an Eulerian graph.

Proof. Suppose that $G$ is a $V_{n}$-Arithmetic graph, where $n=$ $p_{1}^{\alpha_{1}} p_{2}^{\alpha_{2}} \ldots p_{k}^{\alpha_{k}}$. it is well known that the graph $G$ is an Eulerian graph if and only if every vertex of $G$ has an even degree. So,

(1) Let all $\alpha_{i}, i \in\{1,2, \ldots, k\}$ are odd. Then the degrees of the prime vertices $p_{i}, i \in\{1,2, \ldots, k\}$ are odd.

(2) Suppose that, at least one of $\alpha_{i}, i \in\{1,2, \ldots, k\}$ is even call it $\alpha_{r}$ then the vertex $p_{r}$ has an odd degree. 
The Hamiltonian closure of a graph $G$, denoted $C l(G)$, is the simple graph obtained from $G$ by repeatedly adding edges joining pairs of nonadjacent vertices with degree sum at least $|V(G)|$ until no such pair remains. A graph $G$ is Hamiltonian if and only if its closure is Hamiltonian.

THEOREM 7. Let $G$ be a $V_{n}$-Arithmetic graph, where $n=$ $p_{1} p_{2} \ldots p_{k}, 3 \leq k \leq 6$. Then $G$ is a Hamiltonian graph.

Proof. Suppose that $G$ is a $V_{n}$-Arithmetic graph, where $n=$ $p_{1} p_{2} \ldots p_{k}, 3 \leq k \leq 6$. We show that the Hamiltonian closure of $G$ is a complete graph i.e. $C l(G) \cong K_{2^{k}-1}$. and hence, $G$ is Hamiltonian. Let $X_{q}=\left\{u \in V: u=\prod_{i \in B_{q}} p_{i}, B_{q} \subseteq\{1,2, \ldots, k\}\right.$, $\left.\left|B_{q}\right|=q \leq k\right\}$ i.e. $X_{1}=\left\{u=p_{j}: j=1,2, \ldots, k\right\}$, $X_{2}=\left\{u \in V: u=p_{r} p_{s}, r, s=1,2, \ldots, k\right\}, X_{3}=\{u \in$ $\left.V: u=\prod_{i \in B_{3}} p_{i},\left|B_{3}\right|=3\right\}$ and so on.

Step 1. In this step:

(1) The subset of the prime vertices $X_{1}$ will be adjacent to all the vertices of the subset $X_{2}$ because, $\operatorname{deg}\left(p_{j}\right)+\operatorname{deg}\left(p_{r} p_{s}\right)=|V|$ (Corollary 1).

(2) The vertices of $X_{2}$ will be adjacent one to each others because, $\operatorname{deg}\left(p_{j}\right) \leq \operatorname{deg}\left(p_{r} p_{s}\right)$.

So, in the end of this step the degrees of the vertices of $X_{1}$ and $X_{2}$ will become:

(1) $\operatorname{deg}\left(p_{j}\right)=2^{k-1}-1+\left(\begin{array}{c}k-1 \\ 2\end{array}\right)$.

(2) $\operatorname{deg}(u)_{u \in X_{2}}=2^{k-1}+\left(\begin{array}{c}k-2 \\ 1\end{array}\right)+\left(\begin{array}{c}k-2 \\ 2\end{array}\right)$.

Step 2. In this step:

(1) the vertices of $X_{1}$ will be adjacent one to each others because in this case, $\operatorname{deg}\left(p_{j}\right)+\operatorname{deg}\left(p_{r}\right) \geq|V|$. Also, the vertices of $X_{1}$ will be adjacent to all the vertices of $X_{3}$ because,

$\operatorname{deg}\left(p_{j}\right)+\operatorname{deg}(u)_{u \in X_{3}}=7\left(2^{k-3}\right)+\left(\begin{array}{c}k-1 \\ 2\end{array}\right)-1 \geq|V|$, where $3 \leq k \leq 6$.

(2) The vertices of $X_{2}$ will be adjacent to all the vertices of $X_{3}$ because

$\operatorname{deg}(u)_{u \in X_{2}}+\operatorname{deg}(u)_{u \in X_{3}}=7\left(2^{k-3}\right)+\left(\begin{array}{c}k-2 \\ 1\end{array}\right)+\left(\begin{array}{c}k-2 \\ 2\end{array}\right) \geq|V|$, where $3 \leq k \leq 6$.

So, in the end of this step the degrees of the vertices of $X_{1}, X_{2}$ and $X_{3}$ will become:

(1) $\operatorname{deg}\left(p_{j}\right)=2^{k-1}-1+\left(\begin{array}{c}k-1 \\ 1\end{array}\right)+\left(\begin{array}{c}k-1 \\ 2\end{array}\right)+\left(\begin{array}{c}k-1 \\ 3\end{array}\right)$.

(2) $\operatorname{deg}(u)_{u \in X_{2}}=2^{k-1}+2\left(\begin{array}{c}k-2 \\ 1\end{array}\right)+\left(\begin{array}{c}k-2 \\ 2\end{array}\right)+\left(\begin{array}{c}k-2 \\ 3\end{array}\right)$.

(3) $\operatorname{deg}(u)_{u \in X_{3}}=3\left(2^{k-3}\right)+\left(\begin{array}{c}k-3 \\ 1\end{array}\right)+\left(\begin{array}{c}k-3 \\ 2\end{array}\right)+\left(\begin{array}{l}3 \\ 2\end{array}\right)$.

Step3. In this step:

(1) the vertices of $X_{1}$ will be adjacent to all the vertices of $X_{4}$ because,

$\operatorname{deg}\left(p_{j}\right)+\operatorname{deg}(u)_{u \in X_{4}}=3\left(2^{k-2}\right)+\left(\begin{array}{c}k-1 \\ 1\end{array}\right)+\left(\begin{array}{c}k-1 \\ 2\end{array}\right)+\left(\begin{array}{c}k-1 \\ 3\end{array}\right)-$ $1 \geq|V|$, where $4 \leq k \leq 6$.

(2) The vertices of $X_{2}$ will be adjacent to all the vertices of $X_{4}$ because,

$\operatorname{deg}(u)_{u \in X_{2}}+\operatorname{deg}(u)_{u \in X_{4}}=3\left(2^{k-2}\right)+2\left(\begin{array}{c}k-2 \\ 1\end{array}\right)+\left(\begin{array}{c}k-2 \\ 2\end{array}\right)+$ $\left(\begin{array}{c}k-2 \\ 3\end{array}\right) \geq|V|$, where $4 \leq k \leq 6$.

So, in the end of this step the degrees of the vertices of $X_{1}, X_{2}, X_{3}$ and $X_{4}$ will become:

(1) $\operatorname{deg}\left(p_{j}\right)=2^{k-1}-1+\left(\begin{array}{c}k-1 \\ 1\end{array}\right)+\left(\begin{array}{c}k-1 \\ 2\end{array}\right)+\left(\begin{array}{c}k-1 \\ 3\end{array}\right)+\left(\begin{array}{c}k-1 \\ 4\end{array}\right)$.

(2) $\operatorname{deg}(u)_{u \in X_{2}}=2^{k-1}+2\left(\begin{array}{c}k-2 \\ 1\end{array}\right)+2\left(\begin{array}{c}k-2 \\ 2\end{array}\right)+\left(\begin{array}{c}k-2 \\ 3\end{array}\right)+\left(\begin{array}{c}k-2 \\ 4\end{array}\right)$.
(3) $\operatorname{deg}(u)_{u \in X_{3}}=3\left(2^{k-3}\right)+\left(\begin{array}{c}k-3 \\ 1\end{array}\right)+\left(\begin{array}{c}k-3 \\ 2\end{array}\right)+\left(\begin{array}{l}3 \\ 2\end{array}\right)$.

(4) $\operatorname{deg}(u)_{u \in X_{4}}=2^{k-2}+\left(\begin{array}{c}k-4 \\ 1\end{array}\right)+\left(\begin{array}{c}k-4 \\ 2\end{array}\right)+\left(\begin{array}{l}4 \\ 2\end{array}\right)$.

and so on. We observe that:

(1) If $k=3$ then in step $2, C l(G) \cong K_{2^{3}}-1$.

(2) If $k=4$ then in step $4, C l(G) \cong K_{2^{4}}-1$.

(3) If $k=5$ then in step $5, C l(G) \cong K_{2^{5}}-1$.

(4) $k=6$ then in step $6, C l(G) \cong K_{2^{6}}-1$.

Hence, $G$ is Hamiltonian.

Proposition 4. Let $G$ be a $V_{n}$-Arithmetic graph, where $n=p_{1}^{\alpha_{1}} p_{2}^{\alpha_{2}} \ldots p_{k}^{\alpha_{k}}$, such that $\alpha_{i}>1, \forall i \in B$ where $B \subseteq$ $\{1,2, \ldots, k\}$. If $\prod_{i \in B}\left(\alpha_{i}-1\right) \geq k$, then $G$ is not Hamiltonian.

Proof. Let $G$ be a $V_{n}$-Arithmetic graph, where $n=$ $p_{1}^{\alpha_{1}} p_{2}^{\alpha_{2}} \ldots p_{k}^{\alpha_{k}}$, such that $\alpha_{i}>1, \forall i \in B$ where $B \subseteq$ $\{1,2, \ldots, k\}$ and let $V_{0} \subset V$ be the subset of vertices of $V$ whose have degree $k$ i.e. $u \in V_{0} \Leftrightarrow u$ is adjacent only to the prime vertices $p_{i}, i \in\{1,2, \ldots, k\}$. It is clearly that $\left|V_{0}\right|=\prod_{i \in B}\left(\alpha_{i}-1\right)$. Suppose $\left|V_{0}\right| \geq k$. Since $V_{0}$ is an independent set and all $p_{i}$, $i \in\{1,2, \ldots, k\}$ are not adjacent one to each others, then $G$ has no Hamiltonian cycle because if there is a spanning cycle of $G$ then it should pass on at least one vertex twice. So, $G$ is not Hamiltonian.

Let $G$ be a $V_{n}$-Arithmetic graph, where $n=p_{1}^{\alpha_{1}} p_{2}^{\alpha_{2}}$, and $n \neq$ $p_{1} p_{2}$. Then $G$ is a Hamiltonian graph if and only if $1 \leq \alpha_{1}, \alpha_{2} \leq 2$.

Proof. Let $G$ is a $V_{n}$-Arithmetic graph, where $n=p_{1}^{\alpha_{1}} p_{2}^{\alpha_{2}}$, and $n \neq p_{1} p_{2}$. Suppose that $G$ is Hamiltonian and at least $\alpha_{1} \geq 3$. Then there exist at least two vertices $t_{1}, t_{2} \in V$ which are adjacent only to both $p_{1}$ and $p_{2}$. Since $p_{1}, p_{2}$ are not adjacent and $t_{1}, t_{2}$ also are not adjacent, then $G$ has no Hamiltonian cycle. Contradiction.

On the other hand if $1 \leq \alpha_{1}, \alpha_{2} \leq 2$, then we have two cases:

Case 1. If $n=p_{1}^{2} p_{2}^{2}$, then the cycle $\left(p_{1}, p_{1}^{2} p_{2}^{2}, p_{2}, p_{1} p_{2}^{2}, p_{1}^{2}, p_{1} p_{2}, p_{2}^{2}, p_{1}^{2} p_{2}, p_{1}\right)$ in $G$ is a Hamiltonian cycle.

Case 2. If $n=p_{1}^{2} p_{2}$ or $n=p_{1} p_{2}^{2}$, then $G$ has the Hamiltonian cycles $\left(p_{1}, p_{1}^{2} p_{2}, p_{2}, p_{1} p_{2}, p_{1}^{2}, p_{1}\right), \quad\left(p_{2}, p_{1} p_{2}^{2}, p_{1}, p_{1} p_{2}, p_{2}^{2}, p_{2}\right)$ respectively.

So $G$ is Hamiltonian.

\section{M-ARITHMETICAL GRAPHS}

DEFINITION 8. Let $G(V, E)$ be a connected graph with $n$ vertices. Then $G$ is called $m$-Arithmetical graph for some integer $m \geq 1$ if and only if there exists at least one Arithmetic graph $V_{m} \cong G$

\section{EXAMPLE 1.:}

$-P_{3}$ is 6-Arithmetical.

$-K_{2}$ is 4-Arithmetical.

$-S_{n}$ is $2^{n}$-Arithmetical. (where $S_{n}$ is the star with $n$ vertices).

Of course there are infinite Arithmetic graphs $V_{m}$ which is isomorphic to $G$, so, we convince to select the $V_{m}$ where $m$ is the minimum in all cases.

Note that. Since, $V_{m}$ is a connected graph we conclude that all disconnected graphs are not $m$-Arithmetical.

THEOREM 9. Let $G$ be a regular graph. Then $G$ is $m$ Arithmetical graph if and only if $G \cong P_{2}$. 
Proof. Let $G$ be a regular graph. Suppose, $G$ is $m$ Arithmetical graph. Then there is an Arithmetic graph $V_{m}$ such that $V_{m} \cong G$. Then, we have two cases:

Case1. Suppose, $m=p_{1}^{\alpha_{1}} p_{2}^{\alpha_{2}} \ldots p_{k}{ }^{\alpha_{k}}, k \geq 2$. From the proof of Theorem 2.(1) we have

$$
\operatorname{deg}\left(p_{j}\right)-\operatorname{deg}\left(p_{j} p_{l}\right)=-1+\alpha_{l}\left(\alpha_{j}-1\right) \prod_{\substack{i=1 \\ i \neq j, l}}^{k}\left(\alpha_{i}+1\right),
$$

where, $j, l \in\{1,2, \ldots, k\}, j \neq l$.

Let $\alpha_{j}$ be the minimum degree of $p_{i}, i \in\{1,2, \ldots, k\}$. Then

(1) if $\alpha_{j}=1 \Rightarrow \operatorname{deg}\left(p_{j}\right)-\operatorname{deg}\left(p_{j} p_{l}\right)=-1$

which implies that $V_{m}$ is not regular.

(2) if $\alpha_{j}>1$, so, $\alpha_{l} \geq \alpha_{j}>1, \forall l \in\{1,2, \ldots, k\}, l \neq j$, then,

$$
\operatorname{deg}\left(p_{j}\right)-\operatorname{deg}\left(p_{j} p_{l}\right)>0 .
$$

Hence, $V_{m}$, where $m=p_{1}^{\alpha_{1}} p_{2}^{\alpha_{2}} \ldots p_{k}{ }^{\alpha_{k}}, k \geq 2$ is not a regular graph.

Case2. Let $m=p^{\alpha}$. Then, by Theorem 3 .

$$
\Delta\left(V_{m}\right)=\alpha-1, \quad \delta\left(V_{m}\right)=1 .
$$

Since, a graph $G$ is a regular graph if and only if $\Delta(G)=\delta(G)$. Then,

$$
\alpha-1=1 \Rightarrow \alpha=2 .
$$

So, $V_{m}$, where $m=p^{2}$ is a regular graph and hence, $G \cong V_{m} \cong$ $P_{2}$.

The converse is clear.

Let $G$ be a complete graph. Then $G$ is $m$-Arithmetical graph if and only if $G \cong K_{2}$.

THEOREM 10. Let $G$ be a bipartite graph. Then $G$ is $m$ Arithmetical graph if and only if $G$ is a star.

Proof. Let $G$ be a bipartite graph. Suppose, $G$ is $m$ Arithmetical graph. Then there exists an Arithmetic graph $V_{m} \cong$ $G$. We prove that $V_{m}$ is a star. So, we characterize the following cases:

Case1. Suppose, $n=p_{1}^{\alpha_{1}} p_{2}^{\alpha_{2}} \ldots p_{k}{ }^{\alpha_{k}}, k \geq 2$, where at least one of $\alpha_{i} \neq 1, i \in\{1,2, \ldots, k\}$. Then the graph $V_{m}$ has a triangle (an odd cycle subgraph) $\left\langle X_{1}\right\rangle$ where $X_{1}=\left\{p_{j}, p_{j}^{2}, p_{j} p_{l}\right\}$, such that $j, l \in\{1,2, \ldots, k\}, j \neq l$. Hence, $V_{m}$ is not a bipartite graph and so not a star.

Case2. Suppose, $m=p_{1} p_{2} \ldots p_{k}, k \geq 3\left(\alpha_{i}=1\right.$, $\forall i \in\{1,2, \ldots, k\}$ ). In this case the subgraph $\left\langle X_{2}\right\rangle$ of $V_{m}$ where $X_{2}=\left\{p_{r}, p_{r} p_{s}, p_{r} p_{t}\right\}$ is an odd cycle subgraph of $V_{m}$. So, $V_{m}$ is not a star.

Case3. Now it remains that $m=p^{\alpha}$ or $m=p_{1} p_{2}$,

(1) Let $m=p^{\alpha}$. Then all the vertices of $V_{m}$ have a form $p^{a}$, $a \in\{1, \ldots, \alpha\}$. So, the vertex $p$ is adjacent to all the other vertices. Also, the vertices $p^{a}, a \in\{2, \ldots, \alpha\}$ are not adjacent one to each others because, $\operatorname{gcd}\left(p^{a}, p^{b}\right)=p^{a}$ where $a<b$, $a, b \in\{2, \ldots, \alpha\}$. Hence, $V_{m}$ is a star.

(2) Let $m=p_{1} p_{2}$. Then $V=\left\{p_{1}, p_{2}, p_{1} p_{2}\right\}$. The prime vertices $p_{1}, p_{2}$ are not adjacent one to the other and the vertex $p_{1} p_{2}$ is adjacent to both $p_{1}$ and $p_{2}$. Hence, $V_{m}$ is a star.

For the other side, suppose that $G$ is a star. The proof of the first side tell us $V_{m}$ is a star if and only if $m=p^{\alpha}$ or $m=p_{1} p_{2}$ for some positive integer $\alpha$ and some primes $p_{1}, p_{2}$ and $p$. Since, $G=$
$S_{n}$ for some number of vertices $n$, then there exists an Arithmetic graph $V_{m}$ where $m=p^{n}$ such that $V_{m} \cong G=S_{n}$. So, $G$ is $m$-Arithmetical graph.

\section{CONCLUSION}

In this paper, we have studied some of the basic properties of $V_{n}$-Arithmetic graph which will greatly help facilitate the study of many other properties and other parameters for this type of graphs, also they will help to conclude the sufficient and necessary conditions for any graph to be $m$-Arithmetical, which the authors will study it soon.

\section{REFERENCES}

[1] F. Harary, Graph theory, Addison-Wesley, Reading Mass (1969).

[2] M.Manjuri and B.Maheswari, Strong Dominating Sets of Some Arithmetic Graphs. International Journal of Computer Applications 83(3):36-40, December 2013.

[3] M.Manjuri and B.Maheswari, Some Domination Parameters of Arithmetic Vn Graph. International Journal of Computer Applications 92(11):29-32, April 2014.

[4] M.Manjuri and B Maheswari, Strong Dominating Sets of Direct Product Graph of Cayley Graphs with Arithmetic Graphs. International Journal of Applied Information Systems 7(4):1521, June 2014.

[5] Nathanson, Melvyn B. Connected Components of Arithmetic Graphs. Monatshefte fr Mathematik. 1980, Vol. 89, Issue 3, pp. 219-222.

[6] S.Uma Maheswari and B.Maheswari, Some Domination parameters of Arithmetic Graph Vn, IOSR, Journal of Mathematics, Volume 2,Issue 6,(Sep- Oct 2012), 14 -18.

[7] S.Uma Maheswari, B.Maheswari and M.Manjuri, Some Domination Parameters of Direct Product Graphs of Cayley Graphs with Arithmetic Graphs. International Journal of Computer Applications 58(9):32-38, November 2012.

[8] S.Uma Maheswari, B.Maheswari and M.Manjuri, Matching Dominating Sets of Direct Product Graphs of Cayley Graphs with Arithmetic Graphs. International Journal of Computer Applications 60(11):11-16, December 2012.

[9] N.Vasumath. Number theoretic graphs. Ph.D. Thesis, S.V.University, Tirupati, India 1994. 